See discussions, stats, and author profiles for this publication at: https://www.researchgate.net/publication/340993247

\title{
Dynamic Disorder Drives Exciton Transfer in Tubular Chlorosomal Assemblies
}

Article in The Journal of Physical Chemistry B · April 2020

DOI: 10.1021/acs.jpcb.0c00441

CITATIONS

0

4 authors, including:

Xinmeng Li

Leiden University

6 PUBLICATIONS 15 CITATIONS

SEE PROFILE

Huub De Groot

Leiden University

339 PUBLICATIONS 8,104 CITATIONS

SEE PROFILE

Some of the authors of this publication are also working on these related projects:

Artificial water splitting View project

BioSolar Cells View project
Francesco Buda

Leiden University

132 PUBLICATIONS 3,818 CITATIONS

SEE PROFILE 


\title{
Dynamic Disorder Drives Exciton Transfer in Tubular Chlorosomal Assemblies
}

\author{
Xinmeng Li,* Francesco Buda, Huub J. M. de Groot, and G. J. Agur Sevink* \\ Cite This: J. Phys. Chem. B 2020, 124, 4026-4035 \\ Read Online
}

ABSTRACT: Chlorosomes stand out for their highly efficient excitation energy transfer (EET) in extreme low light conditions. Yet, little is known about the EET when a chlorosome is excited to a pure state that is an eigenstate of the exciton Hamiltonian. In this work, we consider the dynamic disorder in the intermolecular electronic coupling explicitly by calculating the electronic coupling terms in the Hamiltonian using nuclear coordinates that are taken from molecular dynamics simulation trajectories. We show that this dynamic disorder is capable of driving the evolution of the exciton, being a stationary state of the initial

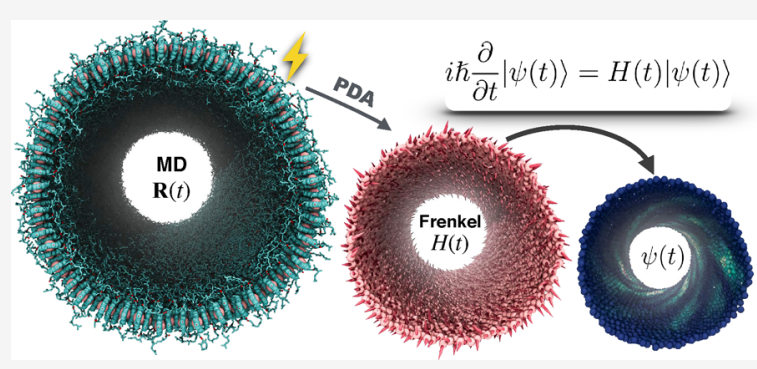
Hamiltonian. In particular, long-distance excitation energy transfer between domains of high exciton population and oscillatory behavior of the population in the site basis are observed, in line with two-dimensional electronic spectroscopy studies. We also found that in the high exciton population domains, their population variation is correlated with their overall coupling strength. Analysis in a reference state basis shows that such dynamic disorder, originating from thermal energy, creates a fluctuating landscape for the exciton and promotes the EET process. We propose such dynamic disorder as an important microscopic origin for the high efficient EET widely observed in different types of chlorosomes, bioinspired tubular aggregates, or other light-harvesting complexes.

\section{INTRODUCTION}

The survival of green sulfur bacteria in extreme low-light condition relies on the efficiency of their light-harvesting antenna, chlorosomes, which are assemblies of hundreds of thousands bacteriochlorophylls (BChls) pigments. ${ }^{1-6}$ Elucidating the design principles behind the efficient excitation energy transfer (EET) that takes place in chlorosomes will contribute to our understanding of photosynthesis ${ }^{7-17}$ and may help to develop artificial light-harvesting ${ }^{18-21}$ or other optoelectronic devices. $^{22-25}$ In spite of recent progress in theoretical ${ }^{26-33}$ and experimental $^{34-40}$ understandings of exciton dynamics in chlorosome systems, a microscopic origin of efficient EET remains unclear.

Previously, we identified a rotational degree freedom that is prominent even though the pigments are densely packed together. ${ }^{41}$ This head-head rotational dynamics between neighboring pigments is expected to affect their electronic couplings. ${ }^{11,42}$ Introducing such rotational disorder in the Frenkel Hamiltonian is found to induce delocalization of exciton states. Optically active states near the bottom of the exciton band are found to be composed of scattered domains of population density that are distributed over the whole tube. ${ }^{42}$ By analyzing the manifold of exciton states along a MD trajectory of molecular conformations, we found level crossings between states and high sensitivity of the exciton state domain patterns to the dynamic disorder. This may induce long-distance exciton transfer between scattered domains involving different exciton states, $^{42}$ in line with $2 \mathrm{D}$ electronic spectroscopy (2DES) studies. ${ }^{37,39}$ Beyond our previous treatment, here we conducted quantum dynamics simulations of exciton evolution, investigate the role of the rotational dynamic disorder on the exciton density matrix, and show that it rapidly converts a pure state generated by optical excitation at the start of the trajectory into a superpositon state characterized by many nonzero off-diagonal elements (a.k.a. coherences) in the density matrix. Considering that such dynamic disorder is also shared by artificial tube assemblies $^{43-45}$ with proven efficient exciton transport ability, we propose thermally induced quantum instability by rapid molecular dynamics, faster than the quantum decoherence, and starting from a pure quantum state as a promising microscopic origin of efficient EET beyond the Born-Oppenheimer regime where the electronic motion follows the nuclear dynamics.

Apart from the previous lack of a precise chlorosome structure, the study of exciton evolution in chlorosomes has been hampered by the large dimension of these assemblies, which rule out any full quantum treatment. ${ }^{46-49}$ In line with previous treatments, ${ }^{26-32,50}$ we therefore consider an effective model, a Frenkel exciton Hamiltonian, as a starting point. Fujita

Received: January 16, 2020

Revised: April 9, 2020

Published: April 28, 2020 
et al. ${ }^{29,31}$ used such a Hamiltonian to study the exciton dynamics of a stacked-ring structure, adding stochastic fluctuations to the site energies, comparing the exciton diffusion under different types of fluctuations. Marquez et al. ${ }^{33}$ studied the exciton dynamics for different lattice models of chlorosomes. They introduced site energy variation through exciton-phonon interactions. Upon exciting a single site in the system, these studies all observed exciton diffusion to the whole tube within a picosecond time. In systems more complicated than a single tube structure, other types of nonstationary initial conditions were also considered. ${ }^{30,32} \mathrm{We}$ note that, however, in such conditions the initial state is a superposition of stationary states, and coherence between the (stationary) exciton states would already be introduced from the start, meaning that phase factors in coherence terms between exciton states of significant energy difference will drive significant exciton evolution in the site basis, even for static Hamiltonians. ${ }^{14,16}$

On the basis of our recent progress in constructing chlorosome structures that satisfy experimental constraints in terms of microscopic detail, ${ }^{41,42,51}$ we are capable of studying the exciton dynamics of realistic chlorosomes within the standard Frenkel exciton treatment. We exploit the nuclear coordinates taken from ground state molecular dynamics trajectories to derive time-dependent electronic coupling terms in the exciton Hamiltonian, thereby including the dynamic disorder explicitly. In line with the low light conditions and in contrast to previous studies, ${ }^{29-33}$ we select the exciton eigenstate of the Hamiltonian with the largest oscillator strength as the initial pure state, i.e., at $t$ $=0$, from which we start our simulation. ${ }^{52}$ Since the initial state is stationary, the evolution of the exciton is fully driven by the dynamic disorder. As a first step toward fully understanding functional mechanisms of chlorosomes, we studied the exciton dynamics in a quasi-closed system manner. While the classical trajectories involve coupling to a heat bath with a thermostat, the propagation is performed in a series of closed quantum system simulations, without including energy dissipation or a sink for the exciton. The dynamics disorder parametrically included in $H(t)$ is able to introduce both the population variation in the state basis and substantial coherences between exciton states on a time scale $<100 \mathrm{fs}$, which we consider sufficiently fast to overcome quantum dephasing and contribute to driving longrange exciton transport.

\section{COMPUTATIONAL METHODS}

Time-Dependent Frenkel Hamiltonian. We conducted all-atom molecular dynamics (MD) simulations to obtain the nuclear coordinates along a trajectory and used these coordinates to extract time-dependent spatial information on the transition dipole vectors $\boldsymbol{\mu}(t)$ and their positions $\mathbf{R}(t)$ (see Figure $S 1$ and computational details in the Supporting Information). Given these spatial properties, the Frenkel Exciton Hamiltonian is calculated by

$$
H(t)=\sum_{i} \nu_{i}|i\rangle\left\langle i\left|+\sum_{i \neq j} J_{i j}(t)\right| j\right\rangle\langle i| \quad i, j \in[1, N]
$$

where diagonal terms $\nu_{i}$ represent the monomer excitation energy of a $\mathrm{BChl} c$ pigment molecule and the off-diagonal terms $J_{i j}$ represent the electronic coupling between molecules $i$ and $j$, which is calculated via the standard point-dipole approximation (PDA)

$$
J_{i j}(t)=\frac{\boldsymbol{\mu}_{i} \cdot \boldsymbol{\mu}_{j}}{\left|\mathbf{R}_{i j}\right|^{3}}-3 \frac{\left(\boldsymbol{\mu}_{i} \cdot \mathbf{R}_{i j}\right)\left(\boldsymbol{\mu}_{j} \cdot \mathbf{R}_{i j}\right)}{\left|\mathbf{R}_{i j}\right|^{5}}
$$

It is known that PDA may overestimate the coupling strengths at distances that are small compared to the spatial dimension of the molecules. ${ }^{53,54}$ Nevertheless, we note that our choice for PDA is noncritical for our treatment and that PDA can be simply replaced by other, possibly more accurate, models when needed. We selected it, in line with most studies of chlorosomes, since it is unique in reproducing the large red shifts in the absorption spectra that are measured in experiments and are due to very strong coupling of $\mathrm{BChl}$ molecules in chlorosomes. ${ }^{42}$

Site Energies. Although chlorosomes exhibit gross structural heterogeneity at the supramolecular level, they are remarkably homogeneous at the molecular level. First, solid state NMR studies on mutant and WT chlorosomes have consistently revealed two well-defined structural fractions. ${ }^{51}$ Second, fluorescence excitation spectroscopy measurements for individual chlorosomes by Günther et al. have confirmed the presence of two well-defined components with very little difference in the average site energies of $\Delta E_{12}=34 \mathrm{~cm}^{-1}$ and limited inhomogeneous broadening. ${ }^{55}$ The energies of the exciton states appear to shift primarily due to variation of the curvature of tubular assemblies. ${ }^{55}$ For a simulated tube of $7.5 \mathrm{~nm}$ radius and with identical site energies for the building blocks, we obtain a total inhomogeneous width of $60 \mathrm{~cm}^{-1}$ due to curvature, which is in line with experimental data that also show static optical broadening in the tubes, while the underlying MAS NMR data reveal high, nearly crystalline homogeneity at the molecular level. ${ }^{55}$ Third, in our earlier work we found converging evidence that the two components are due to molecules with and without interstack hydrogen bonding. ${ }^{41}$ Since the difference in the energies for the lower exciton states are very minor according to the fluorescence excitation data, the direct effect of the environment, including the variation in hydrogen bonding, on the site energies is very small. To simulate these experimentally determined characteristics, the site energies, represented by the diagonal terms in Hamiltonian, were set to a fixed value $\nu_{i}=\nu=15390 \mathrm{~cm}^{-1}$ that is the same for all molecules, while the electronic coupling between molecules (off-diagonal terms in Hamiltonian) are parametrized according to conformations and supramolecular structure taken from MD trajectories, which includes both the molecular rotations and the curvature. More discussions of our choices are available in our previous work. $^{42}$

Exciton Propagation. The evolution of the exciton wave function is governed by the time-dependent Schrödinger equation (TDSE)

$$
\mathrm{i} \hbar \frac{\partial}{\partial t}|\psi(t)\rangle=H(t)|\psi(t)\rangle
$$

and we use an expansion of the $|\psi(t)\rangle$ in terms of site basis $|m\rangle$, corresponding to an exciton that is fully localized at a single molecule of the aggregate:

$$
|\psi(t)\rangle=\sum_{m} c_{m}(t)|m\rangle
$$

Taking use of the orthogonality of the states in site basis $\langle n \mid m\rangle=$ $\delta_{m, n}$ and applying from the left by $\langle n l$, eq 3 is expressed as 


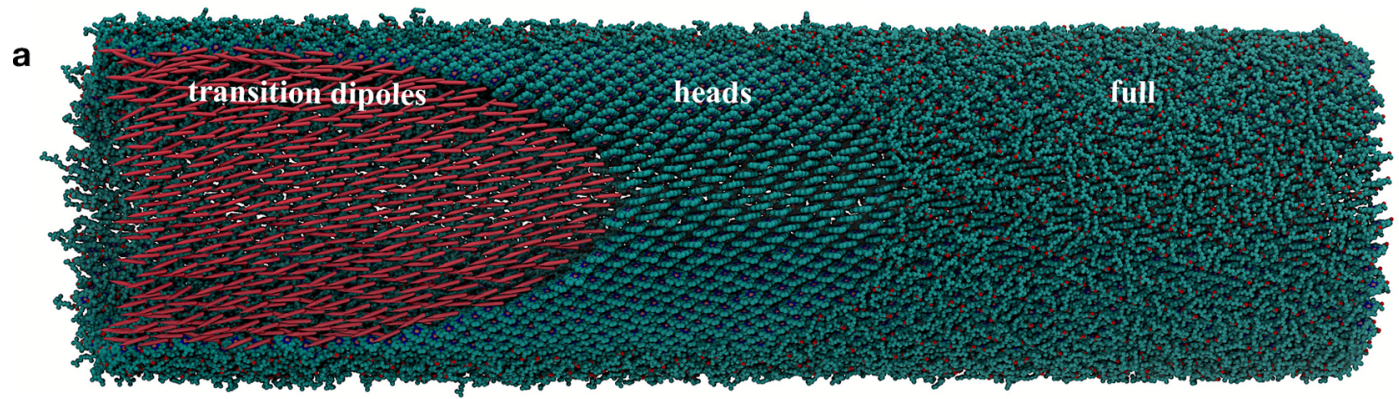

b

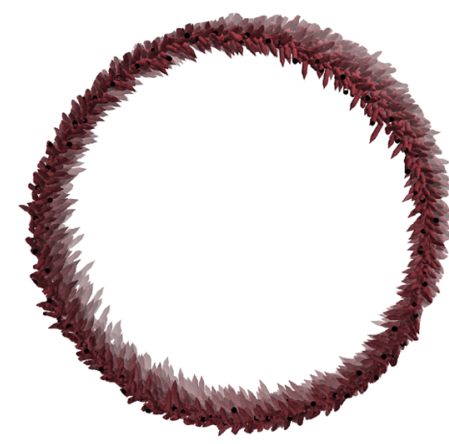

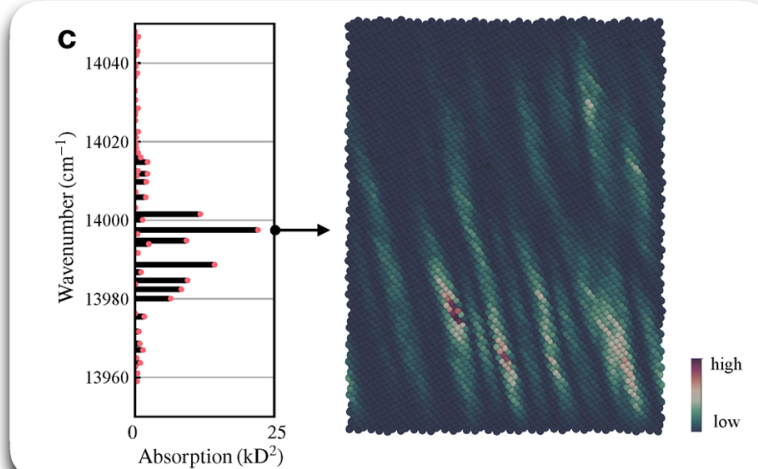

Figure 1. Representative images of the tubular structure and the exciton state of largest oscillator strength for a single chlorosomal tube assembly. (a) Atomistic structure. The tube is composed of $4547 \mathrm{BChl} c$ molecules, with radius $R=7.5 \mathrm{~nm}$ and length $L=60 \mathrm{~nm}$. Transition dipoles and heads of $\mathrm{BChl} c$ molecules are highlighted by removing covering atoms. (b) Orthonormal projection of all the transition dipoles in the whole tube, showing considerable disorder in their relative orientations. (c) Absorption spectrum of the tube (left) and spatial distribution the exciton state of largest oscillator strength (right). The wave function is projected on a plane unrolled from the tube structure; see Figure S2 for other types of visualization.

$$
\mathrm{i} \hbar \dot{c}_{n}(t)=\sum_{m} H_{n m}(t) c_{m}(t)=\nu_{n} c_{n}(t)+\sum_{m}^{m \neq n} J_{n m}(t) c_{m}(t)
$$

Initial Condition. We take the initial wave function $|\psi(0)\rangle$ as $\left|\phi_{M}^{0}\right\rangle$, i.e., the eigenstate of the Hamiltonian $H^{0}=H(t=0)$ with the largest oscillator strength, corresponding to a vertical excitation into the Franck-Condon region:

$$
\begin{aligned}
& H^{0}\left|\phi_{M}^{0}\right\rangle=\varepsilon_{M}\left|\phi_{M}^{0}\right\rangle \\
& \left|\phi_{M}^{0}\right\rangle=\sum_{m} c_{m}^{M}(0)|m\rangle \\
& \nu_{n} c_{n}^{M}(0)+\sum_{m}^{m \neq n} J_{n m}(0) c_{m}^{M}(0)=\varepsilon_{M} c_{m}^{M}(0)
\end{aligned}
$$

Accordingly, we obtained the initial expansion coefficients $c_{m}(0)$ $=c_{m}^{M}(0)$, which are used to solve the evolution of $c_{m}(t)$ eq 5 with a time step of $1 \mathrm{fs}$.

This choice corresponds to a situation in which the chlorosome absorbs one photon in low light conditions. In contrast to previous studies, we particularly did not choose an initial wave function fully localized at one molecular site, because light excites eigenstates rather than individual molecules. ${ }^{52,56}$

Discrete Propagation. Following the numerical integration of the Schrödinger equation (NISE) method, ${ }^{57}$ the timedependent Hamiltonian $H(t)$ is updated discretely every short time interval $\Delta t$ according to the corresponding nuclear positions and is assumed constant in between updates on the $\Delta t$ time intervals. ${ }^{58,59}$ For $j$ th time interval, the constant
Hamiltonian is $H(t=j \Delta t)$, labeled as $H^{j}$, and the individual TDSE we solved is

$$
\begin{aligned}
& \mathrm{i} \hbar \dot{c}_{n}(t)=\sum_{m} H_{n m}^{j} c_{m}(t)=\nu_{n} c_{n}(t)+\sum_{m}^{m \neq n} J_{n m}(j \Delta t) c_{m}(t) \\
& t \in[j \Delta t, j \Delta t+\Delta t]
\end{aligned}
$$

We note that, when the Hamiltonian $H^{j}$ is diagonalized and the corresponding state basis $\left|\phi_{k}^{j}\right\rangle$ is obtained, one can propagate the $|\psi(t)\rangle$ more straightforwardly in the basis $\left|\phi_{k}^{j}\right\rangle$. However, this requires changing of basis and matrix diagonalization for each $\Delta t$, which is slow in our implementation. Instead, we choose to propagate always in site basis through solving the individual TDSE eq 7 using the "zvode" ODE solver in the QUTIP package. ${ }^{60,61}$ More details are available in Scheme S1.

A requirement in such a strategy is that $\Delta t$ is small enough to have a stepwise constant (time-independent) Hamiltonian, meaning that each update only introduces a relatively small perturbation to the Hamiltonian from the previous interval. More specifically, according to eq 5 , it requires that $\Delta t$ is small compared with the time scale of the variation of excitonic couplings $J_{m n}(t)$ on the right-hand side of this equation. Considering the relative motions between molecules are slower than intramolecule vibrational modes, we select $\Delta t=20 \mathrm{fs}$. In particular, we found that the difference in nuclear positions between structures at $t=0$ and $t=20 \mathrm{fs}$ is quite small $(\approx 0.1 \AA)$; see details in later discussions. Furthermore, we take the Hamiltonian $H^{1}=H(t=\Delta t)$ and investigated the exciton dynamics for this constant Hamiltonian, which is referred to as the static case $H^{1}$ in later discussions. As shown later, the exciton evolution in the static case $H^{1}$ is very weak, which 


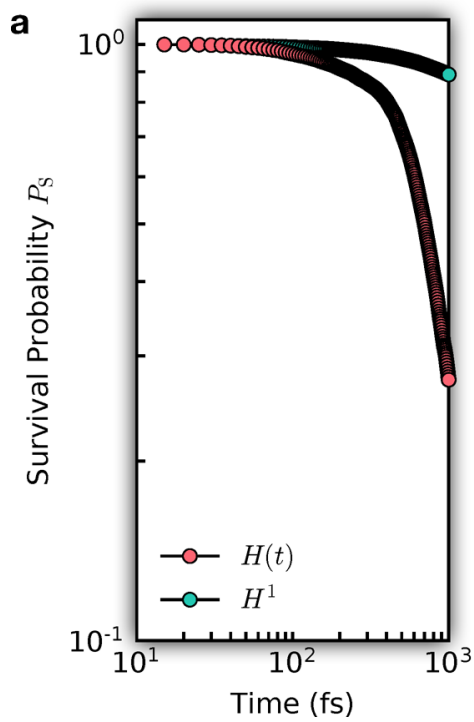

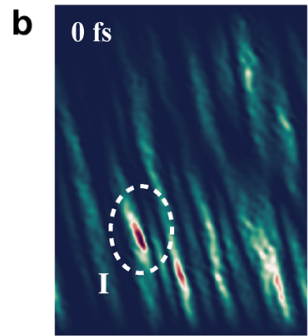

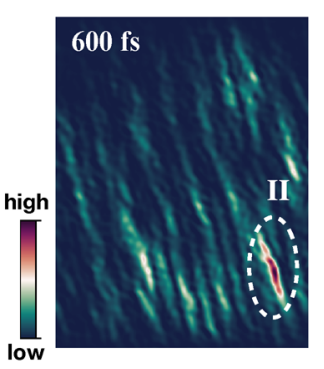

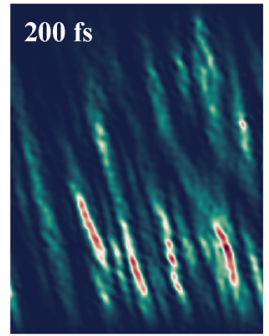
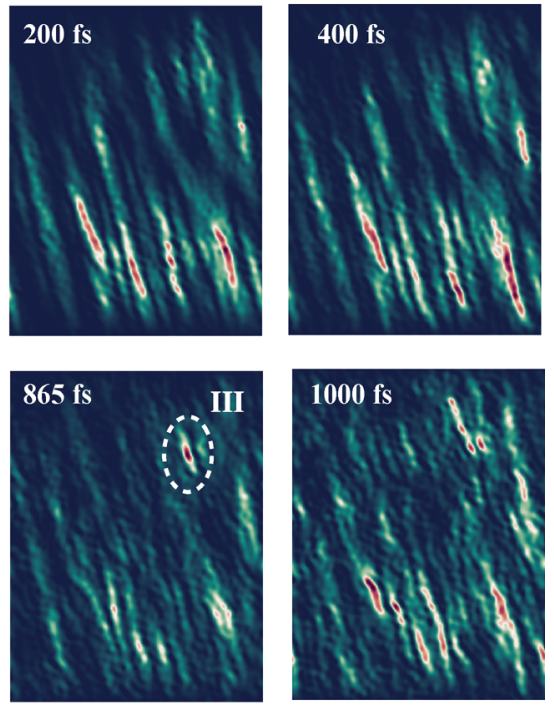

Figure 2. Enhancement of exciton dynamics due to dynamic disorder from MD. (a) Comparison of the survival probability $P_{\mathrm{S}}$ of the initial exciton state in case $H(t)$ and case $H^{1}$. (b) Spatial distribution of the exciton at selected times in the $H(t)$ case. Three domains I-III are labeled to illustrate the longdistance migration. See Movie S1 and Movie S2 for the comparison of whole 1 ps exciton dynamics trajectories in the two cases.

confirms that the perturbation to the Hamiltonian $H^{0}$ is indeed small. We conclude that the $\Delta t=20 \mathrm{fs}$ is small enough in this study. Such an updating strategy is also adopted and discussed in our previous work. ${ }^{42}$

Exciton Dynamics Analysis. The exciton dynamics trajectory is a collection of $|\psi(t)\rangle=\sum_{m} c_{m}(t)|m\rangle$ in site basis; the exciton population/occupation at site $m$ is give by $\left|c_{m}(t)\right|^{2}$. For ease of analysis, we further calculate the corresponding density matrices, which include also coherence information. For any given wave function $|\psi(t)\rangle$, the density matrix $\rho(t)$ in the site basis is obtained as

$$
\rho(t)=|\psi(t)\rangle\left\langle\psi(t)\left|=\sum_{i=1}^{N} \sum_{j=1}^{N} \rho_{i j}(t)\right| i\right\rangle\langle j|
$$

where $\rho_{i j}(t)$ describes the populations $(i=j)$ and coherences $(i \neq$ $j)$ in the site basis. ${ }^{42}$

To clearly characterize the nonstationary nature of the exciton dynamics, we further performed analysis in the reference state basis $\left|\phi_{k}^{0}\right\rangle$, which are the eigenstates of the Hamiltonian at $t=0$, $H_{0}\left|\phi_{k}^{0}\right\rangle=\varepsilon_{k}\left|\phi_{k}^{0}\right\rangle$. The density matrix $\rho^{\phi}$ in $\left|\phi_{k}^{0}\right\rangle$, is calculated from the density matrix in the site basis as

$$
\rho^{\phi}(t)=\mathcal{P}_{0}^{-1} \rho(t) \mathcal{P}_{0}=\sum_{k=1}^{N} \sum_{l=1}^{N} \rho_{k l}^{\phi}(t)\left|\phi_{k}^{0}\right\rangle\left\langle\phi_{l}{ }^{0}\right|
$$

where $\mathcal{P}_{0}$ is the projection matrix composed of the column vectors for states in the state basis $\left|\phi_{k}^{0}\right\rangle$. The density matrix $\rho_{k l}^{\phi}(t)$ now describes the populations $(k=l)$ and coherences $(k \neq l)$ in the reference state basis $\left|\phi_{k}^{0}\right\rangle$.

The eigenstates $\left|\phi_{k}^{j}\right\rangle$ of the Hamiltonian $H^{j}=H(t=j \Delta t)$, with $H^{j}\left|\phi_{k}^{j}\right\rangle=\varepsilon_{k}^{j}\left|\phi_{k}^{j}\right\rangle$, may vary with time. We also calculated the $\rho^{\phi *}(t)$ in such a time-dependent state basis through projecting the density matrix $\rho(t)$ according to the $\left\{\left|\phi_{k}^{j}\right\rangle\right\}$. For the $j$ th time interval:

$$
\begin{aligned}
& \rho^{\phi^{*}}(t)=\mathcal{P}_{j}^{-1} \rho(t) \mathcal{P}_{j}=\sum_{k=1}^{N} \sum_{l=1}^{N} \rho_{k l}^{\phi *}(t)\left|\phi_{k}^{j}\right\rangle\left\langle\phi_{l}^{j}\right| \\
& t \in[j \Delta t, j \Delta t+\Delta t]
\end{aligned}
$$

where the $\mathcal{P}_{j}$ is the projection matrix composed of the column vectors for states $\left|\phi_{k}^{j}\right\rangle$.

\section{RESULTS AND DISCUSSION}

We conducted simulations of the exciton dynamics for both single tube and multitube structures. Most results consider the single tube system, which we used to investigate how the initial eigenstate evolves, and whether long-distance exciton transfer can take place purely driven by dynamic disorder. The multitube system is mainly considered to focus on the effect of hierarchical structure on the intertube exciton transfer.

Single Tube Setup. Figure 1 illustrates relevant details of the tube structure, together with the initial wave function used in the exciton dynamics simulation. Following our static analysis, we selected a $60 \mathrm{~nm}$ long tube with a chiral angle of $49.6^{\circ}$, which matches optical spectra found in experiments. ${ }^{42}$ After equilibration, molecular conformations are collected every $\Delta t$ $=20$ fs along a 1 ps MD trajectory to determine $\{\boldsymbol{\mu}(t), \mathbf{R}(t)\}$ and consequently the Hamiltonian $H(t)$ (eqs 1 and 2). We refer to our previous work for the details about the variation of exciton states in different molecular conformations along such a MD trajectory. ${ }^{42}$ The root-mean-square displacement RMSD that relates to nuclear coordinates that define the transition dipole, shows that the structural deviation from the initial tube continuously grows on the considered time scale of $1 \mathrm{ps}$, whereas the RMSD at $t=20 \mathrm{fs}$ is quite small $(\approx 0.1 \AA)$, indicating that the perturbation $\Delta H$ in $H^{1}=H^{0}+\Delta H$ is indeed quite small; see Figure $\mathrm{S} 4$ for more details. As can be seen from the snapshots in Figure $1 \mathrm{a}, \mathrm{b}$, varying molecular orientations produce considerable rotational disorder in the transition dipoles. We calculated the absorption spectrum for $H^{0}$, and selected the eigenstate with the largest absorption probability (state number $k=20)$ as the initial exciton wave function $|\psi(0)\rangle$ in the exciton dynamics simulation. As Figure $1 c$ shows, disorder in the electronic coupling suppresses exciton delocalization and leads to a few small domains of high exciton population.

Enhancement of Exciton Transport by Dynamic Disorder. In order to quantify transport away from the initial state, we compute the survival probability $P_{S}(t)=|\langle\psi(0) \mid \psi(t)\rangle|^{2}$ 


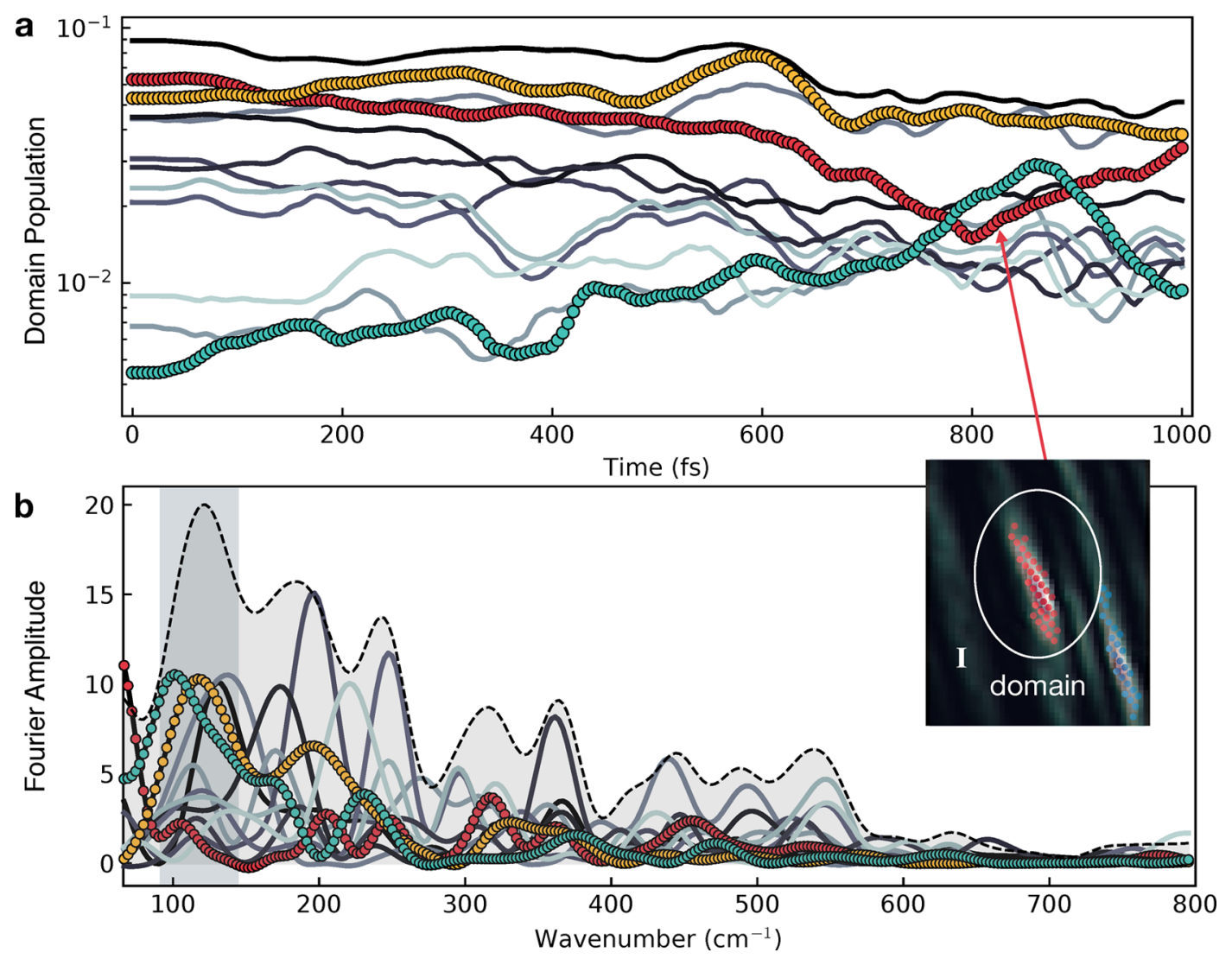

Figure 3. Evolution of the domain population $P_{\text {domain }}(t)$, for distinct domains taken from exciton states at 0,600 , and 865 fs for the $H(t)$ case, showing a clear oscillatory behavior. (a) Domain population along the 1 ps exciton dynamics trajectory. (b) Fourier spectra (amplitude versus wavenumber) calculated from the time traces of the domain populations. The light gray rectangle highlights the frequencies between 91 and $145 \mathrm{~cm}^{-1}$. Domains I, II, and III are highlighted by red, yellow, and green colors. Frequencies corresponding to time scales longer than half of the exciton trajectory of 1 ps are not shown.

of the initial state, ${ }^{58}$ defined as the square of the overlap integral between the initial wave function and the wave function at time $t$; see Figure 2. For the static case, $H^{1}$, the exciton stays close to the original state as $P_{\mathrm{S}}$ is still significant after $1 \mathrm{ps}$, with $P_{\mathrm{S}}>0.9$ along the whole trajectory. For the dynamic case, $H(t), P_{S}$ decreases substantially, with $P_{\mathrm{S}}<0.3$ at 1 ps. The abrupt changing of the power law behavior of $P_{S}$ around $200 \mathrm{fs}$ highlights the role of the dynamic disorder in exciton transfer: the original pure state changes into a mixed state due to the changing Hamiltonian.

For the $H(t)$ case, a significant variation of the spatial distribution of the wave functions is observed, accompanying the decrease of $P_{\mathrm{S}}$, as shown in Figure $2 \mathrm{~b}$ (selected snapshots) and Movie S1. Tracing domains of high exciton population, for instance, the domains I-III labeled in Figure 2b, we observed the presence of (long-distance) exciton energy transfer between such domains. This observation is in line with experimental studies $^{37}$ and our previous static analysis. ${ }^{42}$ The substantial variation of the exciton within the short time of $1 \mathrm{ps}$ is also in line with intrachlorosome exciton transfer time scales (below $1 \mathrm{ps}$ ) observed in experimental works. ${ }^{35-40}$

Our analysis focuses on exciton transport in Hilbert space, which was not considered in previous exciton dynamics studies of chlorosomes and is of importance for understanding the mechanism behind mixing. Qualitative information about the exciton evolution in real space is given in Movies S1 and S2, while Figure S3 provides quantitative information about the mean square displacement of the centers of the exciton. They confirm that, in the $H^{1}$ case, migration in real space is fairly limited, which is consistent with the finding in Hilbert space.

We may relate these observations to photosynthetic bacteria where excitonic energy initially captured in chlorosome antennae need to migrate to the baseplate within a few picoseconds before exciton annihilation occurs. ${ }^{62} \mathrm{We}$ note that during the energy transfer process, a spatial barrier may be present, meaning that the initial state, or a low energy state where an exciton might be trapped, is spatially remote from the target state that is localized at the baseplate. We illustrated such a situation in Scheme S2. In the static case, $H^{1}$, such a spatial barrier will delay or even prohibit the exciton transfer process, as the exciton is trapped in the initial state and the spatial sampling of the exciton is limited (see Movie S2). Introducing dynamic disorder, as shown in the $H(t)$ case, renders the exciton capable of dynamically sampling the whole extent of the tube within the characteristic intrachlorosome transfer time scale of $1 \mathrm{ps}^{62}$ As summarized in Scheme S2, dynamic disorder may introduce "intermediate" domains that are close to the baseplate and helps the following energy transfer.

From the perspective of energy transfer, the exciton dynamics in our $H(t)$ case belongs to a coherent regime, i.e., the regime where energy transfer is perceived as fastest, for which the standard Förster and Redfield theories do not apply. ${ }^{13}$ Such coherent excitation energy transfer (EET) is well-recognized in photosynthetic systems. In such a process, the exciton diffusion equation can be described in terms of a coherent part and an incoherent part. ${ }^{63-65}$ Since our initial exciton state is stationary, 

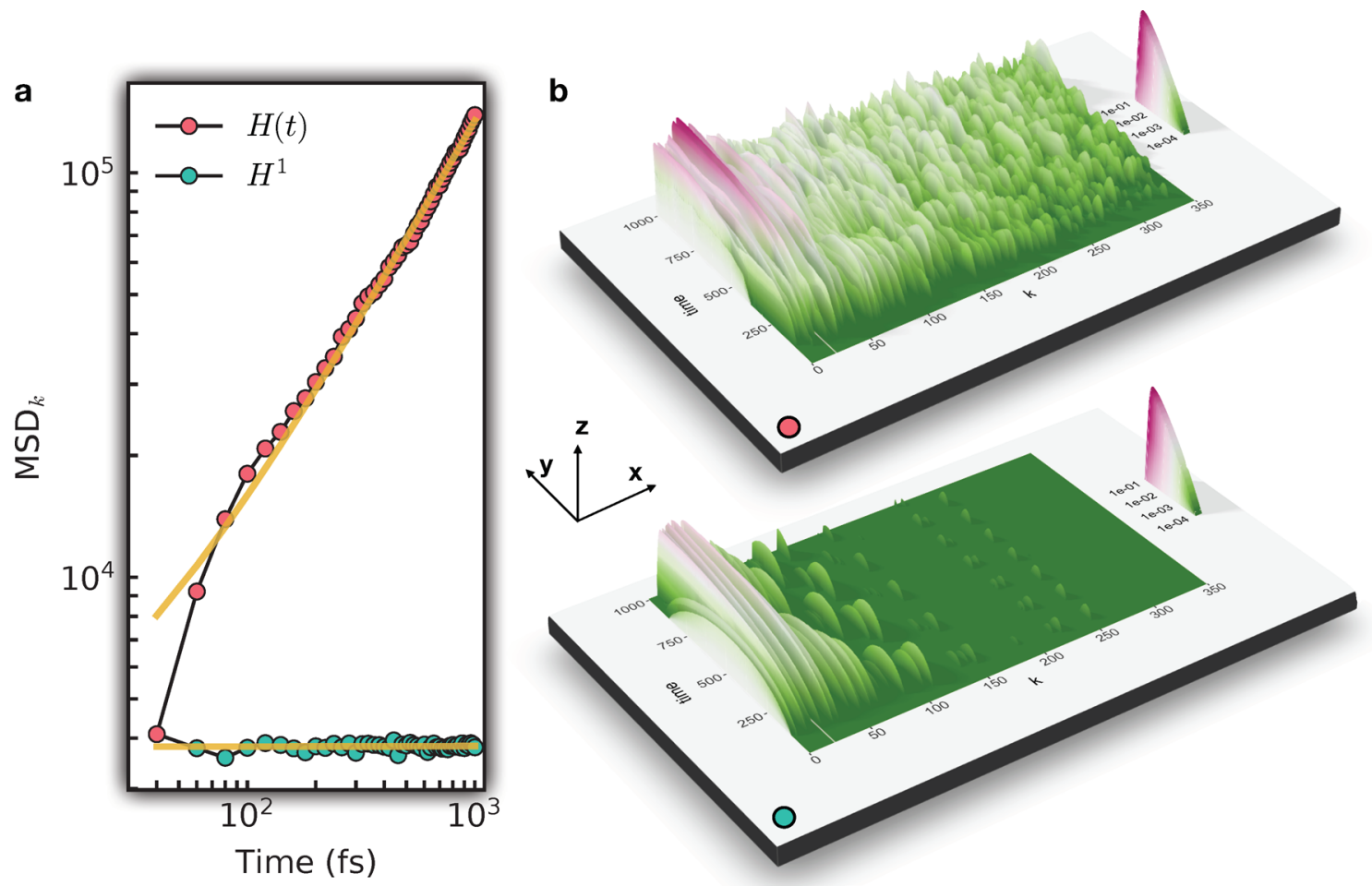

Figure 4. Enhancement of exciton dynamics in state basis due to dynamic disorder obtained from MD. (a) Comparison of the $\mathrm{MSD}_{k}$ in the reference state basis for two cases: the dynamic $H(t)$ and static $H^{1}$. (b) $3 \mathrm{D}$ plots of the population $\rho_{k k}^{\phi}$ in the state basis $\left|\phi_{k}\right\rangle(z$-axis) as a function of state number $k$ $\left(x\right.$-axis) and time $t\left(y\right.$-axis), top for $H(t)$ and bottom for $H^{1}$.

there is initially no coherence between exciton states. Each update of the Hamiltonian $H(t)$ will disturb the coherent evolution in the previous period (incoherent contribution), and at the same time it introduces new coherences (coherent contribution). ${ }^{65}$ This illustrates the complexity of energy transfer in such a coherent regime. ${ }^{13}$

Site Basis Population Modulation. As demonstrated in FMO studies, ${ }^{15,16}$ periodic modulation of the population in the site basis along the exciton dynamics trajectory characterizes quantum beatings that have been detected by $2 \mathrm{DES}$. We investigate the population variation to determine periodic modulations that match coherent beatings measured in chlorosomes systems, e.g., at 91 and $145 \mathrm{~cm}^{-1}$. 39 As domains of high exciton population are formed due to strong electronic coupling between pigments in chlorosomes, we determined the population evolution over domains instead of over individual sites, i.e., via the total population in a domain, $P_{\text {domain }}(t)$ defined as

$$
P_{\text {domain }}(t)=\sum_{\text {sitei } \in \text { domain }} \rho_{i i}(t)
$$

where $\rho_{i i}$ is the population on site $i$. Figure 3 summarizes the $P_{\text {domain }}(t)$ for the considered domains. To capture significant domains at the early and late stages of exciton evolution, we considered domains determined from snapshots at 0,600 , and 865 fs (see Figure $2 b$ ). For clarity, $P_{\text {domain }}(t)$ for the domains labeled I, II, and III in Figure $2 \mathrm{~b}$ are highlighted and more details are provided in the Supporting Information (see Figure S5 for how domains are defined and their $P_{\text {domain }}(t)$ plots).

Oscillatory behavior of the domain population $P_{\text {domain }}$ is observed for all domains (see Figure 3a), which cannot be explained in terms of monomeric properties but suggests many weak coherences along the exciton dynamics trajectory. In particular, the frequency of these oscillations can be quantified by spectral analysis of the $P_{\text {domain }}$ time traces; see Figure $3 \mathrm{~b}$. Concentrating on the range where quantum beats are experimentally observed, i.e., 91 and $145 \mathrm{~cm}^{-1}$ (light gray area in Figure $3 \mathrm{~b}$ ), we find that the average intensity is maximal, with high-population domains (II and III) showing also a maximum intensity. This match between the periodicity of $P_{\text {domain }}$ modulation and experimentally observed coherent beats suggests that quantum beats can also be induced by the ground state nuclear dynamics, in line with the observation in a recent study about FMO. ${ }^{66}$ We note that the $P_{\text {domain }}$ variation in the $H^{1}$ case is much weaker, as shown in Figure S6, and the Fourier spectra are less rich. In particular, the maximum intensity is found around $200 \mathrm{~cm}^{-1}$.

Although the exciton evolution is determined by variation of the Hamiltonian of the whole system, we tried to identify microscopic signatures of the dynamic disorder in the domains that can be correlated to their population oscillations. We first calculated the overall coupling strength $J_{\text {domain }}$, see definition in the Supporting Information. We observe a clear correlation between $J_{\text {domain }}$ and $P_{\text {domain }}$ (Figure S7): higher domain population corresponds to stronger coupling $J_{\text {domain }}$. The calculated cross-correlation values between $J_{\text {domain }}$ and $P_{\text {domain }}$ for the domain I, II, and III are $-0.61,-0.34$, and -0.21 respectively, which shows that they are correlated. This is further supported by the distribution of cross-correlation distribution for all considered domains; see Figure S8a. To see if there is a direct relation between earlier identified simple structural modes, like rotation (average relative rotation angle $\alpha$ ) and lattice vibrations (average distance $d$ between neighboring transition dipoles), and the oscillations of $P_{\text {domain }}$, we analyzed their correlations to $P_{\text {domain }}$ as well; see details in the Supporting Information. We find (see Figure S8) that their cross- 
correlations are dispersed, showing there is no clear direct correlation. Yet, a clear correlation can be identified for $J_{\text {domain }}^{-}$ the negative part of $J_{\text {domain }}$. This indicates that oscillation of domain population cannot be singled out to a particular structural mode but are rather due to collective motion inside the domains.

Density Matrix in State Basis. Features of the nonstationary propagation, such as the variation in population and coherence in the state basis, can be investigated by visualizing the diagonal and off-diagonal terms in the density matrices $\rho^{\phi}(t)$ along the exciton trajectory; see Movie S3. In the movie, we only focus on the $k<350$ part of the density matrix, which covers all the states of significant population: $\rho_{k k}^{\phi}>10 / N(N=4547$, which is the total number of sites; see Figure S9). We refer to Figure S10 for the snapshot at 200 fs and details of our visualization method. Along the exciton dynamics, the exciton mixes from the initial pure state $(k=20)$ with other states in a close energy range. We note that for states $k<350$, the energy range is approximately $350 \mathrm{~cm}^{-1}$. As observed in Movie S3, coherences between the initial state and other states are built, especially in the later stages when dynamic disorder introduces more significant variation to the initial Hamiltonian. As discussed in our previous work, ${ }^{42}$ the eigenstates from $H(t=0)$ to $H(t>0)$ may change. To make sure that the coherences between states in the reference state basis shown in the density matrix $\rho^{\phi}(t)$ is not due to a simple switch of eigenstates, we also analyzed the density matrix $\rho^{\phi *}(t)$ in terms of the state basis of the timedependent Hamiltonian $H(t)$; see details in Scheme S1. As shown in Movie S4, consistent with $\rho^{\phi}(t)$, similar even stronger population variation and introduction of coherences are observed in $\rho^{\phi *}(t)$. In particular, we observed substantial coherences, $\rho_{k l} *(t)$, on a time scale $<100 \mathrm{fs}$; e.g., see $\rho^{\phi *}(t=20$ fs). This indicates that the dynamic disorder in $H(t)$ is sufficient to overcome quantum dephasing and to drive semiclassical exciton transfer. With the population $\rho_{k k}^{\phi *}(t)$, we also calculated the average energy $\bar{E}(t)$ along the exciton dynamics that for the jth time interval,

$$
\bar{E}(t)=\sum_{k=1}^{N} \rho_{k k}^{\phi^{*}}(t) \varepsilon_{k}^{j} \quad t \in[j \Delta t, j \Delta t+\Delta t]
$$

As shown in Figure S11, although $\bar{E}(t)$ slightly increases with time, the variation is within a small range of $1 \mathrm{~kJ} / \mathrm{mol}$. We confirm that there is no artificial increase in the average energy in the evolution process beyond the thermal fluctuation, which is about $2.5 \mathrm{~kJ} / \mathrm{mol}$ at room temperature.

The enhancement of exciton dynamics by dynamic disorder is further demonstrated in the state basis by the analysis of the mean square displacement $\mathrm{MSD}_{k}=\sum_{k} \rho_{k k}(t)\left(k-k_{0}\right)^{2}$ in the reference state basis $\left|\phi_{k}\right\rangle$. Using $\mathrm{MSD}_{k} \propto D t$, the extracted diffusion coefficient $D$ in the $H(t)$ case, $D_{t}=21.983 \mathrm{fs}^{-1}$, is about 4 orders of magnitude larger than $D_{1}=0.002 \mathrm{fs}^{-1}$ for $H^{1}$; see Figure $4 \mathrm{a}$. Consistent with our analysis of the exciton dynamics trajectories in real space, exciton transport in state basis is limited for the static $H^{1}$ case, where the exciton state is trapped in the initial state. In the dynamic $H(t)$ case, however, exciton transport in state basis is facilitated by the dynamic disorder that stems from the thermal energy.

Landscape. We may further analyze these results by considering the time traces of populations in the reference state basis for all states $k<350$, via the $3 \mathrm{D}$ plots in Figure $4 \mathrm{~b}$. From the $H(t)$ case, Figure $4 \mathrm{~b}$ top, it is clear that thermal energy creates a fluctuating (potential) energy landscape for the exciton, which drives migration in the states basis and enhances mixing of exciton states. Without accumulation of perturbations due to thermal fluctuation, in the $H^{1}$ case (Figure $4 \mathrm{~b}$ bottom), the exciton dynamics is much simpler, with well-defined oscillations resulting from fixed phase factors in the coherence terms. Animations for different perspectives of the $3 \mathrm{D}$ plots for the two cases are given in Movie S5 and Movie S6.

Multitube. As experimental imaging shows that chlorosomes consist of hierarchical tube structures, ${ }^{51}$ we also conducted exciton dynamics simulations on two types of hierarchical assemblies: concentric tubes ( 3 nested tubes) and side-by-side aligned tubes ( 2 tubes). We focused on the question how the intertube connectivity affects the exciton transfer. Figure S12 shows simulation details and our analysis of the initial state survival probability $P_{\mathrm{S}}$ as well as the tube population $P_{\text {tube }}(t)=\sum_{\text {sitei tube }} \rho_{i i}(t)$ along the exciton trajectories. As characterized by $P_{\mathrm{S}}$, the enhancement of exciton transfer by dynamic disorder is also present in both the multitube cases. By comparing $P_{\text {tube }}$ for both multitube topologies, we find stronger intertube fluctuations in the concentric tube case and $P_{\text {tube }}$ reaches equilibrium faster, reflecting a stronger intertube electronic coupling in the concentric tube topology. The intertube transfer time scale, i.e., the time that $P_{\text {tube }}$ reaches equilibrium, is about $4 \mathrm{ps}$ (concentric tube) to $10 \mathrm{ps}$ (aligned tubes), which is longer than the intratube transfer time scale of 1 ps. This result suggests that prior to energy annihilation at a time scale of $10 \mathrm{ps},{ }^{62}$ an exciton is able to migrate across different layers in concentric tubes and across separate tubes.

For the different tube radii $R$ in the concentric tube case, the equilibrium $P_{\text {tube }}$ increases with increasing radius $R$, in line with our previous static analysis that there is a biased transfer from inner to outer tube due to the geometric condition that larger tubes contain more molecules with $N \propto R^{2} .^{42}$ In particular, when syn-anti packing units assemble into tubes, their hydrophobic farnesyl tails will cover both the inside and outside of each nested tube, which provides the option of forming tightly packed concentric tubes. ${ }^{41}$ Compared with the side-by-side tube topology, the intertube exciton dynamics is enhanced and biased toward to the outer tube in the concentric tube topology, which is more favored in terms of the efficiency in the whole energy transfer process.

How can we relate our computational results to natural chlorosomes? In nature, chlorosomes are oblong-shaped assemblies of complete and incomplete concentric tubes, with an overall dimension that is generally smaller than the wavelength of the absorbed light. ${ }^{87,68}$ It therefore makes sense to assume that incident light will excite an eigenstate that is delocalized over the entire chlorosome (see the results in this subsection), albeit that high exciton populations will again be confined to small domains that are scattered over the whole structure. In particular, since the total population in each of the concentric tubes is found to scale with the number of constituting pigments at longer times, we may conclude that the intratube excitonic features are rather independent of the tube radius. Thus, they are likely to agree with that of a single tube.

The EET, however, can be seen to depend on the intertube connectivity, with a more efficient exciton transfer in concentric than in side-by-side tubular assemblies. Yet, since chlorosomes are known to assemble via a nucleation and growth process, ${ }^{69}$ it is unlikely that such functional requirements prescribe the actual tube connectivity in natural chlorosomes. It is more likely that 
energy transfer to a "sink" like the baseplate starts directly after excitation, and that mixing of exciton states due to the dynamic disorder from nuclear motions plays a more dominant role in EET than the actual intertube connectivity.

\section{CONCLUSION}

The essential requirement for utilizing thermal energy to enhance the exciton transfer is that the strength of dynamic disorder, associated with thermal motions, is comparable to the energy gaps between exciton states. ${ }^{13}$ If the energy gaps are too large or the dynamic disorder too weak, migration in the state basis will be limited, as has been illustrated for the static case $H^{1}$. Liquid-crystalline materials composed of densely packed pigments, for instance, chlorosomes and carbocyanine dye nanotubes, are suitable for high-efficient long-distance EET since these requirements are easily met: (1) The presence of a large number of pigments expressing structural disorder guarantees a large number of exciton states of very similar energy. (2) The relative low energetic costs of pigment rotation in the tube due to thermal motion, even in dense packing, gives rise to rotation of transition dipoles or dynamic disorder. This is in contrast to the situation where pigments are confined in a protein matrix, where disorder mainly stems from the environment in which pigments are trapped. (3) The fluctuating nature of the dynamic disorder, at several lengths and time scales, prevents trapping in eigenstates, for instance low energy states, and promotes efficient exciton transfer, in line with a recent 2DES study. ${ }^{40}$

By including the pigment assembly dynamics stemming from ground state molecular dynamics at room temperature in the fluctuating electronic coupling terms of our Frenkel Hamiltonian, we have been able to study the role of dynamic disorder in enhancing the exciton transfer. We observed several phenomena that are in line with experimental observations, despite the simplifications considered in our approach. Since the coupling calculation based on PDA considers only three atoms in a BChl molecule (see Figure S1), one could assume the electronic coupling $J(t)$ to be more sensitive to the nuclear position variation along the nuclear trajectory. We anticipate that methodological improvements, ${ }^{47,57}$ for instance, introducing more precise methods for the electronic coupling as well as the earlier mentioned site-dependent site energy, will bring a stronger dynamics disorder in the $H(t)$ and strengthen our main conclusions. Considering that a minimal system of chlorosomes is composed of $\sim 5000 \mathrm{BChl}$ pigments ( $\sim 0.5$ million atoms), accurate parametrization of site energies fluctuations following dynamically varying nuclear positions $\mathbf{R}(t)$, for instance via $a b$ initio MD simulation and TDDFT calculation, is unrealistic. The conventional treatment, i.e., including fluctuations in the diagonal terms without addressing the dynamics of the underlying molecules, has the clear disadvantage of blurring or even destroying correlated fluctuations that are included in the coupling terms via MD. However, our work paves the way for more accurately including the limited variation of site energies in a next step, and this is outside the scope of the present study. If the splitting between the two components is on the order of collective vibrations, this may give rise to additional vibrionic couplings and level crossings for further enhancing the effects discussed in our manuscript. In particular, it will be interesting to see how very slow site energy variations, which will be averaged on the NMR time scale and appear as static heterogeneity on the optical time scale, can interact with fluctuations in the coupling terms to play a role in exciton dynamics. One may anticipate them to induce additional transient off-diagonal coupling terms, which will then have to take into account the two defining characteristics of the chlorosome system: strong coupling between $\mathrm{BChl}$, and the time variation of the coupling strength.

In chlorosomes, local molecular motion is a key property. It is a generic property for structures composed of a head-head packing unit and independent of the particular molecular component (or BChl pigment), the hierarchical nature of the structure (close/open tubular or planar), or overall size. Simple but general, dynamic disorder that originates from these local molecular motions is capable of inducing a fluctuating landscape for the excitons, which enhances exciton transfer. Our findings are a key step toward solving the long-lasting puzzle about why efficient exciton transfer is widely observed in different types of chlorosomes and other light-harvesting complexes. Following this principle, we also suggest that the liquid-crystal-like aggregates assembled from pigments with some sort of symmetry breaking, for instance, chlorosomes and the carbocyanine dye nanotubes, all rely on the same principle for efficient long-distance energy transfer.

\section{ASSOCIATED CONTENT}

\section{Supporting Information}

The Supporting Information is available free of charge at https://pubs.acs.org/doi/10.1021/acs.jpcb.0c00441.

Extra computational details; schematic representation of the exciton dynamics simulation setup; different types of spatial visualization of the wave function; RMSD of molecular conformations along the 1 ps MD trajectory; schematic representation of a possible spatial barrier in energy transfer process; identification of domains in the tube and their exciton population variation for the case of $H(t)$ and the case of $H^{1}$; comparison and correlation analysis of different domain properties for the case of $H(t)$; collection of states in the reference state; visualization of the density matrix; average energy of the exciton; exciton dynamics results for two multitube structures with different intertube topologies (PDF)

Visualization of the exciton population in the site basis along the 1 ps exciton dynamics trajectory for the case of $H(t)$ (MP4)

Visualization of the exciton population in the site basis along the 1 ps exciton dynamics trajectory for the case of of $H^{1}$ (MP4)

Visualization of the density matrix $\rho^{\phi}$ in the reference state basis of $H^{0}$ along the 1 ps exciton dynamics trajectory for the case of $H(t)$ (MP4)

Visualization of the density matrix $\rho^{\phi *}$ in the state basis of time-dependent $H(t)$ along the 1 ps exciton dynamics trajectory for the case of $H(t)$ (MP4)

Different perspectives (animated) of the 3D plot of the time traces of populations $\rho_{k k}^{\phi}(t)$ for the case of $H(t)$ (MP4)

Different perspectives (animated) of the 3D plot of the time traces of populations $\rho_{k k}^{\phi}(t)$ for the case of $H^{1}$ (MP4)

\section{AUTHOR INFORMATION}

\section{Corresponding Authors}

Xinmeng Li - Leiden University, Leiden Institute of Chemistry, 2300 RA Leiden, The Netherlands; 10 orcid.org/0000-00026863-6078; Email: x.li@lic.leidenuniv.nl 
G. J. Agur Sevink - Leiden University, Leiden Institute of Chemistry, 2300 RA Leiden, The Netherlands; (ㄱ) orcid.org/ 0000-0001-8005-0697; Email: a.sevink@chem.leidenuniv.nl

\author{
Authors \\ Francesco Buda - Leiden University, Leiden Institute of \\ Chemistry, 2300 RA Leiden, The Netherlands; (1) orcid.org/ \\ 0000-0002-7157-7654 \\ Huub J. M. de Groot - Leiden University, Leiden Institute of \\ Chemistry, 2300 RA Leiden, The Netherlands
}

Complete contact information is available at:

https://pubs.acs.org/10.1021/acs.jpcb.0c00441

\section{Notes}

The authors declare no competing financial interest.

\section{ACKNOWLEDGMENTS}

The authors acknowledge Prof. dr. A. R. Holzwarth for helpful discussions. The use of supercomputer facilities was sponsored by NWO Exact and Natural Sciences, with financial support from The Netherlands Organization for Scientific Research (NWO).

\section{REFERENCES}

(1) Overmann, J.; Cypionka, H.; Pfennig, N. An Extremely Low-light Adapted Phototrophic Sulfur Bacterium From the Black Sea. Limnol. Oceanogr. 1992, 37, 150-155.

(2) Beatty, J. T.; Overmann, J.; Lince, M. T.; Manske, A. K.; Lang, A. S.; Blankenship, R. E.; Van Dover, C. L.; Martinson, T. A.; Plumley, F. G. An Obligately Photosynthetic Bacterial Anaerobe From a Deep-Sea Hydrothermal Vent. Proc. Natl. Acad. Sci. U. S. A. 2005, 102, 93069310.

(3) Olson, J. M. Chlorophyll Organization and Function in Green Photosynthetic Bacteria. Photochem. Photobiol. 1998, 67, 61-75.

(4) Pšenčík, J.; Arellano, J. B.; Ikonen, T. P.; Borrego, C. M.; Laurinmäki, P. A.; Butcher, S. J.; Serimaa, R. E.; Tuma, R. Internal Structure of Chlorosomes From Brown-Colored Chlorobium Species and the Role of Carotenoids in Their Assembly. Biophys. J. 2006, 91, $1433-1440$

(5) Oostergetel, G. T.; Van Amerongen, H.; Boekema, E. J. The Chlorosome: A Prototype for Efficient Light Harvesting in Photosynthesis. Photosynth. Res. 2010, 104, 245-255.

(6) Orf, G. S.; Blankenship, R. E. Chlorosome Antenna Complexes From Green Photosynthetic Bacteria. Photosynth. Res. 2013, 116, 315331.

(7) Hohmann-Marriott, M. F.; Blankenship, R. E. Evolution of Photosynthesis. Annu. Rev. Plant Biol. 2011, 62, 515-548.

(8) Scholes, G. D.; Fleming, G. R.; Olaya-Castro, A.; Van Grondelle, R. Lessons From Nature About Solar Light Harvesting. Nat. Chem. 2011, 3, 763-774.

(9) Croce, R.; Van Amerongen, H. Natural Strategies for Photosynthetic Light Harvesting. Nat. Chem. Biol. 2014, 10, 492-501.

(10) Bryant, D. A.; Canniffe, D. P. How Nature Designs LightHarvesting Antenna Systems: Design Principles and Functional Realization in Chlorophototrophic Prokaryotes. J. Phys. B: At., Mol. Opt. Phys. 2018, 51, 033001.

(11) Pavel, M.; Rienk, v. G. Interplay of Disorder and Delocalization in Photosynthetic Light Harvesting. Curr. Opin. Chem. Biol. 2018, 47, 16.

(12) Fassioli, F.; Dinshaw, R.; Arpin, P. C.; Scholes, G. D. Photosynthetic Light Harvesting: Excitons and Coherence. J. R. Soc., Interface 2014, 11, 20130901.

(13) Chenu, A.; Scholes, G. D. Coherence in Energy Transfer and Photosynthesis. Annu. Rev. Phys. Chem. 2015, 66, 69-96.
(14) Rebentrost, P.; Mohseni, M.; Kassal, I.; Lloyd, S.; Aspuru-Guzik, A. Environment-Assisted Quantum Transport. New J. Phys. 2009, 11, 033003.

(15) Ishizaki, A.; Fleming, G. R. Theoretical Examination of Quantum Coherence in a Photosynthetic System At Physiological Temperature. Proc. Natl. Acad. Sci. U. S. A. 2009, 106, 17255-17260.

(16) Panitchayangkoon, G.; Hayes, D.; Fransted, K. A.; Caram, J. R.; Harel, E.; Wen, J.; Blankenship, R. E.; Engel, G. S. Long-Lived Quantum Coherence in Photosynthetic Complexes At Physiological Temperature. Proc. Natl. Acad. Sci. U. S. A. 2010, 107, 12766-12770.

(17) Duan, H.; Prokhorenko, V. I.; Cogdell, R. J.; Ashraf, K.; Stevens, A. L.; Thorwart, M.; Miller, R. J. D. Nature Does Not Rely on LongLived Electronic Quantum Coherence for Photosynthetic Energy Transfer. Proc. Natl. Acad. Sci. U. S. A. 2017, 114, 8493-8498.

(18) Balaban, T. S. Tailoring Porphyrins and Chlorins for SelfAssembly in Biomimetic Artificial Antenna Systems. Acc. Chem. Res. 2005, 38, 612-623.

(19) Miyatake, T.; Tamiaki, H. Self-Aggregates of Natural Chlorophylls and Their Synthetic Analogues in Aqueous Media for Making Light-Harvesting Systems. Coord. Chem. Rev. 2010, 254, 25932602.

(20) Hiramoto, M.; Kitada, K.; Iketaki, K.; Kaji, T. Near Infrared Light Driven Organic P-I-n Solar Cells Incorporating Phthalocyanine JAggregate. Appl. Phys. Lett. 2011, 98, 023302.

(21) Eisele, D. M.; Cone, C. W.; Bloemsma, E. A.; Vlaming, S. M.; Van Der Kwaak, C. G. F.; Silbey, R. J.; Bawendi, M. G.; Knoester, J.; Rabe, J. P.; Vanden Bout, D. A. Utilizing Redox-Chemistry to Elucidate the Nature of Exciton Transitions in Supramolecular Dye Nanotubes. Nat. Chem. 2012, 4, 655-662.

(22) Grosso, G.; Graves, J.; Hammack, A. T.; High, A. A.; Butov, L. V.; Hanson, M.; Gossard, A. C. Excitonic Switches Operating At Around 100 K. Nat. Photonics 2009, 3, 577-580.

(23) Modesto-Lopez, L. B.; Thimsen, E. J.; Collins, A. M.; Blankenship, R. E.; Biswas, P. Electrospray-Assisted Characterization and Deposition of Chlorosomes to Fabricate a Biomimetic LightHarvesting Device. Energy Environ. Sci. 2010, 3, 216-222.

(24) Akselrod, G. M.; Walker, B. J.; Tisdale, W. A.; Bawendi, M. G.; Bulovic, V. Twenty-Fold Enhancement of Molecular Fluorescence By Coupling to a J-Aggregate Critically Coupled Resonator. ACS Nano 2012, 6, 467-471.

(25) Harmatys, K. M.; Chen, J.; Charron, D. M.; Maclaughlin, C. M.; Zheng, G. Multipronged Biomimetic Approach to Create Optically Tunable Nanoparticles. Angew. Chem., Int. Ed. 2018, 57, 8125-8129.

(26) Didraga, C.; Klugkist, J. A.; Knoester, J. Optical Properties of Helical Cylindrical Molecular Aggregates: The Homogeneous Limit. J. Phys. Chem. B 2002, 106, 11474-11486.

(27) Prokhorenko, V. I.; Steensgaard, D. B.; Holzwarth, A. R. Exciton Theory for Supramolecular Chlorosomal Aggregates: 1. Aggregate Size Dependence of the Linear Spectra. Biophys. J. 2003, 85, 3173-3186.

(28) Linnanto, J. M.; Korppi-Tommola, J. E. L. Exciton Description of Chlorosome to Baseplate Excitation Energy Transfer in Filamentous Anoxygenic Phototrophs and Green Sulfur Bacteria. J. Phys. Chem. B 2013, 117, 11144-11161.

(29) Fujita, T.; Brookes, J. C.; Saikin, S. K.; Aspuru-Guzik, A. Memory-Assisted Exciton Diffusion in the Chlorosome Light-Harvesting Antenna of Green Sulfur Bacteria. J. Phys. Chem. Lett. 2012, 3, 2357-2361.

(30) Huh, J.; Saikin, S. K.; Brookes, J. C.; Valleau, S.; Fujita, T.; Aspuru-Guzik, A. Atomistic Study of Energy Funneling in the LightHarvesting Complex of Green Sulfur Bacteria. J. Am. Chem. Soc. 2014, 136, 2048-2057.

(31) Fujita, T.; Huh, J.; Saikin, S. K.; Brookes, J. C.; Aspuru-Guzik, A. Theoretical Characterization of Excitation Energy Transfer in Chlorosome Light-Harvesting Antennae From Green Sulfur Bacteria. Photosynth. Res. 2014, 120, 273-289.

(32) Sawaya, N. P. D.; Huh, J.; Fujita, T.; Saikin, S. K.; Aspuru-Guzik, A. Fast Delocalization Leads to Robust Long-Range Excitonic Transfer in a Large Quantum Chlorosome Model. Nano Lett. 2015, 15, 17221729. 
(33) Marquez, A. S.; Chen, L.; Sun, K.; Zhao, Y. Probing Ultrafast Excitation Energy Transfer of the Chlorosome With Exciton-Phonon Variational Dynamics. Phys. Chem. Chem. Phys. 2016, 18, 2029820311

(34) Psencik, J.; Polivka, T.; Nemec, P.; Dian, J.; Kudrna, J.; Maly, P.; Hala, J. Fast Energy Transfer and Exciton Dynamics in Chlorosomes of the Green Sulfur Bacterium Chlorobium Tepidum. J. Phys. Chem. A 1998, 102, 4392-4398.

(35) Prokhorenko, V. I.; Steensgaard, D. B.; Holzwarth, A. R. Exciton Dynamics in the Chlorosomal Antennae of the Green Bacteria Chloroflexus Aurantiacus and Chlorobium Tepidum. Biophys. J. 2000, 79, 2105-2120.

(36) Martiskainen, J.; Linnanto, J.; Aumanen, V.; Myllyperkio, P.; Korppi-Tommola, J. Excitation Energy Transfer in Isolated Chlorosomes From Chlorobaculum Tepidum and Prosthecochloris Aestuarii. Photochem. Photobiol. 2012, 88, 675-683.

(37) Dostál, J.; Mančal, T.; Augulis, R.; Vácha, F.; Pšenčík, J.; Zigmantas, D. Two-Dimensional Electronic Spectroscopy Reveals Ultrafast Energy Diffusion in Chlorosomes. J. Am. Chem. Soc. 2012, 134, 11611-11617.

(38) Jun, S.; Yang, C.; Isaji, M.; Tamiaki, H.; Kim, J.; Ihee, H. Coherent Oscillations in Chlorosome Elucidated By Two-Dimensional Electronic Spectroscopy. J. Phys. Chem. Lett. 2014, 5, 1386-1392.

(39) Dostal, J.; Mancal, T.; Vacha, F.; Psencik, J.; Zigmantas, D. Unraveling the Nature of Coherent Beatings in Chlorosomes. J. Chem. Phys. 2014, 140, 115103.

(40) Jun, S.; Yang, C.; Kim, T. W.; Isaji, M.; Tamiaki, H.; Ihee, H.; Kim, J. Role of Thermal Excitation in Ultrafast Energy Transfer in Chlorosomes Revealed By Two-Dimensional Electronic Spectroscopy. Phys. Chem. Chem. Phys. 2015, 17, 17872-17879.

(41) Li, X.; Buda, F.; De Groot, H. J. M.; Sevink, G. J. A. Contrasting Modes of Self-Assembly and Hydrogen-Bonding Heterogeneity in Chlorosomes of Chlorobaculum Tepidum. J. Phys. Chem. C 2018, 122, 14877-14888.

(42) Li, X.; Buda, F.; De Groot, H. J. M.; Sevink, G. J. A. Molecular Insight in the Optical Response of Tubular Chlorosomal Assemblies. J. Phys. Chem. C 2019, 123, 16462-16478.

(43) Eisele, D. M.; Arias, D. H.; Fu, X.; Bloemsma, E. A.; Steiner, C. P.; Jensen, R. A.; Rebentrost, P.; Eisele, H.; Tokmakoff, A.; Lloyd, S.; et al. Robust Excitons Inhabit Soft Supramolecular Nanotubes. Proc. Natl. Acad. Sci. U. S. A. 2014, 111, E3367-E3375.

(44) Caram, J. R.; Doria, S.; Eisele, D. M.; Freyria, F. S.; Sinclair, T. S.; Rebentrost, P.; Lloyd, S.; Bawendi, M. G. Room-Temperature MicronScale Exciton Migration in a Stabilized Emissive Molecular Aggregate. Nano Lett. 2016, 16, 6808-6815.

(45) Cui, Q. H.; Peng, Q.; Luo, Y.; Jiang, Y.; Yan, Y.; Wei, C.; Shuai, Z.; Sun, C.; Yao, J.; Zhao, Y. S. Asymmetric Photon Transport in Organic Semiconductor Nanowires Through Electrically Controlled Exciton Diffusion. Sci. Adv. 2018, 4, No. eaap9861.

(46) Renger, T. Theory of Excitation Energy Transfer: From Structure to Function. Photosynth. Res. 2009, 102, 471-485.

(47) González, L.; Escudero, D.; Serrano-Andrés, L. Progress and Challenges in the Calculation of Electronic Excited States. ChemPhysChem 2012, 13, 28-51.

(48) Cupellini, L.; Jurinovich, S.; Campetella, M.; Caprasecca, S.; Guido, C. A.; Kelly, S. M.; Gardiner, A. T.; Cogdell, R.; Mennucci, B. An $\mathrm{Ab}$ Initio Description of the Excitonic Properties of Lh2 and Their Temperature Dependence. J. Phys. Chem. B 2016, 120, 11348-11359.

(49) Curutchet, C.; Mennucci, B. Quantum Chemical Studies of Light Harvesting. Chem. Rev. 2017, 117, 294-343.

(50) Brixner, T.; Hildner, R.; Koehler, J.; Lambert, C.; Wuerthner, F. Exciton Transport in Molecular Aggregates - From Natural Antennas to Synthetic Chromophore Systems. Adv. Energy Mater. 2017, 7, 1700236.

(51) Ganapathy, S.; Oostergetel, G. T.; Wawrzyniak, P. K.; Reus, M.; Chew, A. G. M.; Buda, F.; Boekema, E. J.; Bryant, D. A.; Holzwarth, A. R.; De Groot, H. J. M. Alternating Syn-Anti Bacteriochlorophylls Form Concentric Helical Nanotubes in Chlorosomes. Proc. Natl. Acad. Sci. U. S. A. 2009, 106, 8525-8530.
(52) Chenu, A.; Maly, P.; Mancal, T. Dynamic Coherence in Excitonic Molecular Complexes Under Various Excitation Conditions. Chem. Phys. 2014, 439, 100-110.

(53) Madjet, M.; Abdurahman, A.; Renger, T. Intermolecular Coulomb Couplings From $\mathrm{Ab}$ Initio Electrostatic Potentials: Application to Optical Transitions of Strongly Coupled Pigments in Photosynthetic Antennae and Reaction Centers. J. Phys. Chem. B 2006, 110, 17268-17281.

(54) Kenny, E. P.; Kassal, I. Benchmarking Calculations of Excitonic Couplings Between Bacteriochlorophylls. J. Phys. Chem. B 2016, 120, $25-32$.

(55) Günther, L. M.; Jendrny, M.; Bloemsma, E. A.; Tank, M.; Oostergetel, G. T.; Bryant, D. A.; Knoester, J.; Köhler, J. Structure of Light-Harvesting Aggregates in Individual Chlorosomes. J. Phys. Chem. B 2016, 120, 5367-5376.

(56) Fassioli, F.; Olaya-Castro, A.; Scholes, G. D. Coherent Energy Transfer Under Incoherent Light Conditions. J. Phys. Chem. Lett. 2012, 3, 3136-3142.

(57) Bondarenko, A. S.; Knoester, J.; Jansen, T. L. C. Comparison of Methods to Study Excitation Energy Transfer in Molecular Multichromophoric Systems. Chem. Phys. 2020, 529, 110478.

(58) Monti, A.; Negre, C. F. A.; Batista, V. S.; Rego, L. G. C.; De Groot, H. J. M.; Buda, F. Crucial Role of Nuclear Dynamics for Electron Injection in a Dye-Semiconductor Complex. J. Phys. Chem. Lett. 2015, 6, 2393-2398.

(59) Shi, L.; Willard, A. P. Modeling the Effects of Molecular Disorder on the Properties of Frenkel Excitons in Organic Molecular Semiconductors. J. Chem. Phys. 2018, 149, 094110.

(60) Brown, P. N.; Byrne, G. D.; Hindmarsh, A. C. Vode: A VariableCoefficient Ode Solver. SIAM J. Sci. Comput. 1989, 10, 1038-1051.

(61) Johansson, J. R.; Nation, P. D.; Nori, F. Qutip 2: A Python Framework for the Dynamics of Open Quantum Systems. Comput. Phys. Commun. 2013, 184, 1234-1240.

(62) Dostal, J.; Psencik, J.; Zigmantas, D. In Situ Mapping of the Energy Flow Through the Entire Photosynthetic Apparatus. Nat. Chem. 2016, 8, 705-710.

(63) Haken, H.; Reineker, P. The Coupled Coherent and Incoherent Motion of Excitons and Its Influence on the Line Shape of Optical Absorption. Eur. Phys. J. A 1972, 249, 253-268.

(64) Haken, H.; Strobl, G. An Exactly Solvable Model for Coherent and Incoherent Exciton Motion. Z. Phys. A: Hadrons Nucl. 1973, 262, $135-148$.

(65) Huijser, A.; Savenije, T. J.; Meskers, S. C. J.; Vermeulen, M. J. W.; Siebbeles, L. D. A. The Mechanism of Long-Range Exciton Diffusion in a Nematically Organized Porphyrin Layer. J. Am. Chem. Soc. 2008, 130, 12496-12500.

(66) Tempelaar, R.; Jansen, T. L. C.; Knoester, J. Vibrational Beatings Conceal Evidence of Electronic Coherence in the Fmo LightHarvesting Complex. J. Phys. Chem. B 2014, 118, 12865-12872.

(67) Staehelin, L. A.; Golecki, J. R.; Drews, G. Supramolecular Organization of Chlorosomes (Chlorobium Vesicles) and of Their Membrane Attachment Sites in Chlorobium Limicola. Biochim. Biophys. Acta, Bioenerg. 1980, 589, 30-45.

(68) Adams, P. G.; Cadby, A. J.; Robinson, B.; Tsukatani, Y.; Tank, M.; Wen, J.; Blankenship, R. E.; Bryant, D. A.; Hunter, C. N. Comparison of the Physical Characteristics of Chlorosomes From Three Different Phyla of Green Phototrophic Bacteria. Biochim. Biophys. Acta, Bioenerg. 2013, 1827, 1235-1244.

(69) Balaban, T. S.; Leitich, J.; Holzwarth, A. R.; Schaffner, K. Autocatalyzed Self-Aggregation of (3(1)r)-[et,et]bacteriochlorophyll $\mathrm{C}(\mathrm{f})$ Molecules in Nonpolar Solvents. Analysis of the Kinetics. J. Phys. Chem. B 2000, 104, 1362-1372. 